D

Dept of Infectious Diseases, Aarhus University Hospital, Aarhus, Denmark.

\title{
Bridging for lung transplantation with lumacaftor/ivacaftor
}

\section{Case report}

Cite as: Pedersen SS, Jensen-Fangel $S$,

Jeppesen M. Bridging for lung transplantation with lumacaftor/ivacaftor. Breathe 2018; 14: e68-e71.

\section{Introduction}

Cystic fibrosis (CF) is a multi-organ disease caused by mutations in the cystic fibrosis transmembrane regulator (CFTR) gene, with consequent dysfunction of the CFTR channel. The most common mutation in CF is delta F508. CFTR-modulating therapy with lumacaftor/ivacaftor corrects (lumacaftor) the misfolded CFTR channel and potentiates (ivacaftor) its function at the cell surface [1].

Task 1

What is the impact of a malfunctioning CFTR channel in the lungs? 


\section{Answer 1}

Lack of chloride transport through the CFTR channel causes a thick and motionless layer of mucus in the lungs. This impairs the removal of bacteria, fungi and viruses leading to chronic lung infections and inflammation [2].

Several studies have investigated the effects and side-effects of these new treatments [3]. In these studies, primary and secondary end-points have been changes in forced expiratory volume in $1 \mathrm{~s}\left(\mathrm{FEV}_{1}\right) \%$ predicted, incidence of pulmonary exacerbation, changes in body mass index (BMI) and respiratory symptoms. Lumacaftor/ivacaftor has resulted in a significant, although limited increase in $\mathrm{FEV}_{1} \%$ predicted in patients homozygous for the delta F508 mutation with variable results regarding the effects on BMI [4].
In this case report, we demonstrate the impact of lumacaftor/ivacaftor on routine inflammatory markers and on the general condition in a CF patient with end-stage lung disease waiting for a lung transplant.

\section{Case history}

A 21-year-old female with CF, homozygous for the delta F508 mutation and pancreas insufficient, had been diagnosed with CF at the age of 15 months due to failure to thrive. The patient had chronic lung infection with Staphylococcus aureus, Stenotrophomonas maltophilia and Aspergillus fumigatus. She did not have CF-related diabetes or CF-related liver disease. After considerable pulmonary instability and declining lung function she was accepted onto the lung transplant waiting list in October 2013.

\section{Task 2}

When should patients with CF be considered for lung transplantation? 

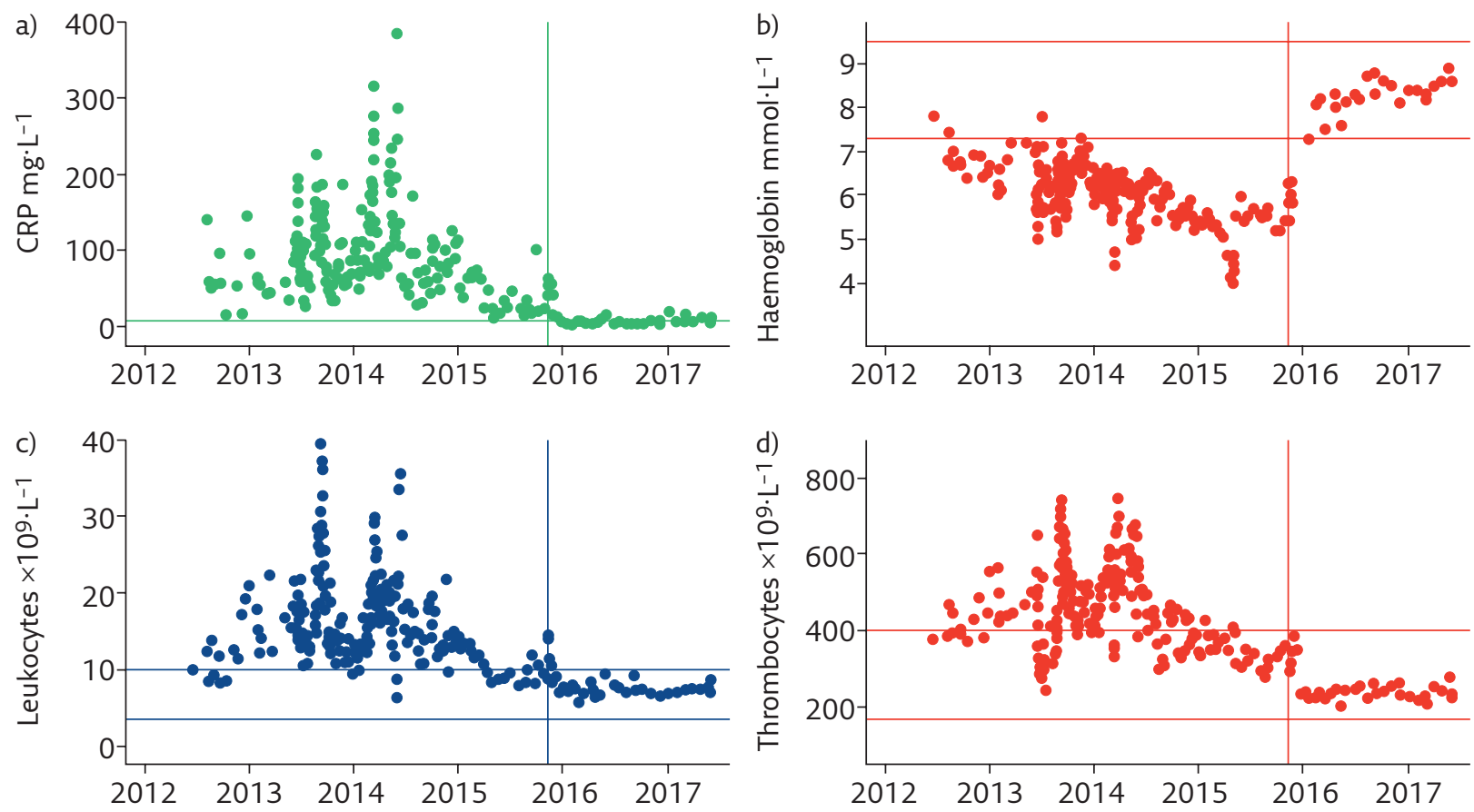

Figure 1 Scatter plots of a) CRP, b) haemoglobin, c) leukocytes and d) thrombocytes over time. Horizontal lines represent the reference ranges for each parameter. Vertical lines indicate the onset of treatment with lumacaftor/ivacaftor. Labels on the $x$-axis indicate the start of each year.

\section{Answer 2}

- $\mathrm{FEV} 1$ of $\leq 30 \%$ predicted or a rapid decline in younger patients.

- Oxygen therapy for hypoxaemia.

- Frequent pulmonary exacerbations that respond poorly to treatment [5].

In November 2015 she began treatment with lumacaftor/ivacaftor. Prior to initiation of treatment with lumacaftor/ivacaftor $\mathrm{FEV} 1$ was $0.59 \mathrm{~L}(20 \%$ predicted) and BMI was $16.9 \mathrm{~kg} \cdot \mathrm{m}^{-2}$.

For comparison we analysed the mean values of haemoglobin, platelet count and inflammatory markers (C-reactive protein (CRP) and leukocyte counts) 5 years prior to lung transplantation. We also investigated the effect of lumacaftor/ ivacaftor on the 6-min walk test (6MWT). This test was performed once every 3 months in the department of respiratory medicine, while the patient was on the waiting list for a lung transplant. Data analysis was performed using STATA 13 (StataCorp LP, College Station, TX, USA; 2013). All end-points were categorised into two groups: before and during treatment with lumacaftor/ivacaftor.

All four parameters changed significantly (figure 1). The inflammation parameter CRP decreased significantly after starting lumacaftor/ ivacaftor from a mean of $99.08 \mathrm{mg} \cdot \mathrm{L}^{-1}(95 \% \mathrm{Cl}$ : 91.31-106.86) to $8.24 \mathrm{mg} \cdot \mathrm{L}^{-1}(95 \% \mathrm{Cl}: 7.56-$ 23.89) $(p<0.001)$ and leukocyte counts decreased significantly from a mean of $16.49 \times 10^{9} \cdot \mathrm{L}^{-1}(95 \% \mathrm{Cl}$ : $15.72-17.28)$ to $8.24 \times 10^{9} \cdot \mathrm{L}^{-1}(95 \% \mathrm{Cl}: 7.45-9.03)$ $(\mathrm{p}<0.001)$. Figure $1 \mathrm{a}$ and $\mathrm{c}$ illustrates a stabilisation towards the point of initiation of lumacaftor/ivacaftor treatment. In addition, both parameters had a transitory increase after the onset of treatment.

Task 3

Should chronic lung inflammation be treated with corticosteroids? 


\section{Answer 3}

Corticosteroids have no effect in patients with CF. Chronic maintenance therapy consists of:

- Mucolytics, which degrade mucus, improve lung function and reduce the frequency of pulmonary exacerbation;

- Hydrator therapy, which hydrates airway surfaces improving lung function and reducing the frequency of pulmonary exacerbation; and

- Antibiotic therapy, for the eradication or suppression of bacterial infections [5].

Haemoglobin increased significantly from a mean of $6.01 \mathrm{mmol} \cdot \mathrm{L}^{-1}$ (95\% Cl: 5.93-6.09) to $7.74 \mathrm{mmol} \cdot \mathrm{L}^{-1}(95 \% \mathrm{Cl}: 7.34-8.13)(\mathrm{p}<0.001)$. Figure $1 \mathrm{~b}$ illustrates the increase in haemoglobin after a steady drop in the time prior to treatment. High levels of thrombocytes from reactive thrombocytosis decreased significantly from a mean of $466.3 \times 10^{9} \cdot \mathrm{L}^{-1}$ (95\% Cl: $452.2-480.6)$ to $261.3 \times 10^{9} \cdot \mathrm{L}^{-1}(95 \% \mathrm{Cl}$ : 243.7-278.9). The patient did not receive blood transfusions during the course of treatment.

Following treatment, $\mathrm{FEV} 1$ at week 26 was $0.55 \mathrm{~L}$ (18\% predicted) and BMI was $17.4 \mathrm{~kg} \cdot \mathrm{m}^{-2}$. At week 55, FEV1 was $0.65 \mathrm{~L}$ (21\% predicted) and BMI was $17.6 \mathrm{~kg} \cdot \mathrm{m}^{-2}$.

The patient experienced considerable changes in her general condition after starting lumacaftor/ ivacaftor treatment. The patient's need for oxygen therapy stopped and, from being mostly immobilised using wheelchair, she regained walking ability, improving her 6MWT from a mean of $246.4 \mathrm{~m}$ (95\% Cl: $198.5-294.4)$ to a mean of $374.6 \mathrm{~m}$ (95\% Cl: $307.5-441.7)(p=0.002)$. She had social interaction with friends, which had not taken place for several years.

In June 2017, after 3.5 years on the waiting list, the patient had a lung transplant.

\section{Discussion}

We report the case of a young female CF patient homozygous for delta F508 and with terminal respiratory insufficiency, who started treatment with lumacaftor/ivacaftor and experienced a significant reduction in inflammatory markers and a significant increase in haemoglobin. These changes led to a significant improvement of physical ability and of quality of life; however, the patient did not experience an increase in FEV1 after starting therapy due to irreversible lung damage. Ultimately, treatment with lumacaftor/ivacaftor was essential in bridging to lung transplantation. Early effects of lumacaftor/ivacaftor have been demonstrated with respiratory events [6]. Our data on leukocytes and CRP support these findings. Our findings from blood samples indicate new positive outcomes, which should be investigated in larger studies.

\section{Conflict of interest}

None declared.

\section{References}

1. Cutting GR. Cystic fibrosis genetics: from molecular understanding to clinical application. Nat Rev Genet 2015; 16: 45-56.

2. Smith AL, Redding G, Doershuk C, et al. Sputum changes associated with therapy for endobronchial exacerbation in cystic fibrosis. J Pediatr 1988; 112: 547-554.

3. Wainwright CE, Elborn JS, Ramsey BW. Lumacaftor-ivacaftor in patients with cystic fibrosis homozygous for Phe508del CFTR. N EnglJ Med 2015; 373: 1783-1784.

4. Elborn JS, Ramsey BW, Boyle MP, et al. Efficacy and safety of lumacaftor/ivacaftor combination therapy in patients with cystic fibrosis homozygous for Phe508del CFTR by pulmonary function subgroup: a pooled analysis. Lancet Respir Med 2016; 4: 617-626.

5. Smyth AR, Bell SC, Bojcin S, et al. European Cystic Fibrosis Society standards of care: best practice guidelines. J Cyst Fibros 2014; 13: Suppl. 1, S23-S42.

6. Popowicz N, Wood J, Tai A, et al. Immediate effects of lumacaftor/ivacaftor administration on lung function in patients with severe cystic fibrosis lung disease. J Cyst Fibros 2017; 16: 392-394 